TITLE:

\title{
Generator coordinate method with a conjugate momentum: application to the particle number projection
}

\section{AUTHOR(S):}

Hizawa, N.; Hagino, K.; Yoshida, K.

\section{CITATION:}

Hizawa, N. ... [et al]. Generator coordinate method with a conjugate momentum:

application to the particle number projection. Physical Review C 2021, 103(3): 034313.

ISSUE DATE:

2021-03

URL:

http://hdl.handle.net/2433/268036

RIGHT:

(c) 2021 American Physical Society 


\title{
Generator coordinate method with a conjugate momentum: Application to particle number projection
}

\author{
N. Hizawa $\odot$, K. Hagino $\odot$, and K. Yoshida $\odot$ \\ Department of Physics, Kyoto University, Kyoto 606-8502, Japan
}

(Received 21 December 2020; accepted 1 March 2021; published 17 March 2021)

\begin{abstract}
We discuss an extension of the generator coordinate method (GCM) by taking simultaneously a collective coordinate and its conjugate momentum as generator coordinates. To this end, we follow the idea of the dynamical GCM (DGCM) proposed by Goeke and Reinhard. We first show that the DGCM method can be regarded as an extension of the double projection method for the center of mass motion. As an application of DGCM, we then investigate the particle number projection, for which we not only carry out an integral over the gauge angle as in the usual particle number projection but also take a linear superposition of Barden-Cooper-Schrieffer (BCS) states which have different mean particle numbers. We show that the ground state energy is significantly lowered by such effect, especially for magic nuclei for which the pairing gap is zero in the BCS approximation. This suggests that the present method makes a good alternative to the VAP method, as the method is much simpler than the VAP.
\end{abstract}

DOI: 10.1103/PhysRevC.103.034313

\section{INTRODUCTION}

Beyond mean-field calculations based on the generator coordinate method (GCM) have been rapidly developing in recent years [1-25]. In this method, the wave function of many-body states is described as a linear superposition of many Slater determinants [26]. In this way, quantum correlations beyond the mean field approximation are incorporated in the ground-state wave function. Furthermore, the GCM provides not only the ground state but also excited states described by the chosen generator coordinates. For these reasons, the GCM has often been employed for a microscopic description of nuclear collective motions.

While the idea of GCM is conceptually simple, it has also long been known that a naive GCM cannot describe properly the center of motion of a nucleus. That is, the appropriate moment of inertia associated with the translational motion, i.e., the total mass of a system, cannot be obtained by simply superposing the wave functions located at different center of mass positions [26]. Peierls and Thouless resolved this problem by projecting the GCM state on a state with a definite linear momentum [27]. This method has been referred to as the double projection method, which has also been formulated for a rotational motion [27].

Recently, the idea based on the double projection method has been put forward by Borrajo et al. [28,29] as well as by Shimada et al. [30-32] for a calculation of rotational bands in deformed nuclei. These authors employed crankedHartree-Fock-Bogoliubov states with several deformations and rotational frequencies as basis states for the GCM calculations after performing the angular momentum projection. By including the cranked states with a broken time-reversal symmetry, it was shown that the excitation energies in the rotational bands are significantly lowered.
One can view these results form a different point of view. That is, the angular momentum projection is carried out by superposing many-body states with different orientation angles of the principle axes. To this end, the angular momentum projection is usually applied to the states with a single rotational frequency only. Superposing cranked states with different rotational frequencies implies that the quantity conjugate to the angle, that is, the angular momentum, is incorporated in GCM states. This suggests that one can achieve a better description of collective states by simultaneously treating a collective coordinate and its conjugate momentum in the GCM method.

In addition to the double projection method, there are several ways to extend the GCM along this line, such as the complex GCM, in which collective coordinates are regarded as complex numbers $[26,33,34]$. In this connection, we mention that Goeke and Reinhard have formulated the GCM by introducing the conjugate momentum apart from a collective variable and called it the dynamical GCM (DGCM) [35-39]. It has been argued that the DGCM includes the complex GCM as a special case and that the DGCM is an extension of the double projection method. However, due to its complexity, no concrete numerical calculations have been carried out with DGCM as far as the authors know.

The aim of this paper is to apply the DGCM to the particle number fluctuation in a BCS wave function, for which a pair of the canonical variables is known a priori, that is, the gauge angle and the particle number. Treating these variables as generator coordinates is nothing but an application of the DGCM. This amounts to superposing many BCS states with different particle numbers after performing the particle number projection, as in Refs. [28-32] for rotational motions. This work can in fact be regarded as the first step in a long-range project of applications of the DGCM to nuclear collective motions. The fact that there is only a single variable (and its conjugate) 
for the collective coordinate is another numerical advantage to investigating the particle number fluctuation.

The paper is organized as follows. In Sec. II, we give a brief review of the DGCM. We then show that the DGCM is equivalent to a generalization of the double projection method when a constraint operator is considered. We discuss specific cases of quantum number projections for the angular momentum, the momentum of the center of mass, and the particle number. In Sec. III, we apply the DGCM to BCS calculations and discuss the effect of a fluctuation of mean particle numbers on the ground state of spherical nuclei. We then summarize the paper and discuss future perspectives in Sec. IV.

\section{DYNAMICAL GCM AND GENERALIZATION OF THE DOUBLE PROJECTION METHOD}

\section{A. A brief summary of DGCM}

In the GCM, one diagonalizes a Hamiltonian $\hat{H}$ in the space spanned by states $\{|\boldsymbol{q}\rangle\}$ which are parametrized by generator coordinates $\boldsymbol{q}$. Usually, many-body Slater determinants are used for the states $\{|\boldsymbol{q}\rangle\}$ with a real number $\boldsymbol{q}$. Notice that the states $\{|\boldsymbol{q}\rangle\}$ are not orthogonal to each other. For simplicity, in the following, we consider only a single generator coordinate, $q$. A many-body wave function is then expanded as

$$
|\psi\rangle=\int d q f(q)|q\rangle
$$

In this equation, the weight function $f(q)$ is determined by the variational principle, which leads to the Hill-Wheeler equation [26],

$$
\int d q^{\prime}\left(\left\langle q|\hat{H}| q^{\prime}\right\rangle-E\left\langle q \mid q^{\prime}\right\rangle\right) f\left(q^{\prime}\right)=0
$$

where $E$ is an energy eigenvalue. $\left\langle q|\hat{H}| q^{\prime}\right\rangle$ and $\left\langle q \mid q^{\prime}\right\rangle$ are referred to as the Hamiltonian and the overlap kernels, respectively. The GCM is often employed to describe collective motions, and in this sense $q$ is called a collective coordinate.

In principle, if one could generate a collective coordinate properly, the GCM could correctly describe a collective motion. This is the case, e.g., for a system described by the Lipkin model [26]. However, in general, it is an extremely difficult problem to find properly a collective path, and one often determines it in an empirical way. There is no guarantee that the basis constructed in this way adequately takes into account the relevant dynamics of a collective motion which one wants to describe.

In order to overcome this problem, Goeke and Reinhard have extended the GCM by introducing the canonical momentum $p$ conjugate to the collective coordinate $q$ and defined the basis states which satisfy

$$
\left\langle q, p\left|\overleftarrow{\partial}_{q} \vec{\partial}_{p}-\overleftarrow{\partial}_{p} \vec{\partial}_{q}\right| q, p\right\rangle=i
$$

Here, $\overleftarrow{\partial}_{q}$ and $\vec{\partial}_{q}$ act on the left-hand and the right-hand sides of $q$, respectively, and similar for $\overleftarrow{\partial}_{p}$ and $\vec{\partial}_{p}$. Notice that we have set $\hbar=1$. The path connecting $|q, p\rangle$ is called a dynamical path.
The condition (3) can also be written in a form of the commutation relation,

$$
\left\langle q, p\left|\left[\hat{Q}_{0}, \hat{P}_{0}\right]\right| q, p\right\rangle=i,
$$

where $\hat{Q}_{0}$ and $\hat{P}_{0}$ are generators of $q$ and $p$ defined as

$$
\begin{gathered}
\hat{Q}_{0}|q, p\rangle=-\left(i \partial_{p}+\frac{\partial S}{\partial p}\right)|q, p\rangle, \\
\hat{P}_{0}|q, p\rangle=\left(i \partial_{q}+\frac{\partial S}{\partial q}\right)|q, p\rangle,
\end{gathered}
$$

respectively, with an arbitrary smooth function, $S=S(q, p)$, of $q$ and $p$. The function $S=S(q, p)$ originates from the freedom to choose any phase of the state $|q, p\rangle$. In Ref. [39], the phase was chosen so that the expectation values of the two operators $\hat{Q}_{0}$ and $\hat{P}_{0}$ are zero.

After the dynamical path is somehow obtained, one can expand a wave function using the states $|q, p\rangle$ as

$$
|\psi\rangle=\iint d q d p f(q, p)|q, p\rangle .
$$

This is called the dynamical GCM (DGCM) [35-39]. The weight function $f(q, p)$ is determined by solving the HillWheeler equation, as in the usual GCM.

In general, not all the states specified by the two parameters $q$ and $p$ contribute to a collective motion. For example, if one could find a function $\Gamma\left(q, p ; q^{\prime}\right)$ which satisfies

$$
|q, p\rangle=\int d q^{\prime} \Gamma\left(q, p ; q^{\prime}\right)\left|q^{\prime}, 0\right\rangle
$$

for an arbitral pair of $(q, p)$, the wave function in the DGCM, Eq. (7), is reduced to the wave function in the GCM, Eq. (1), with

$$
f_{\mathrm{GCM}}\left(q^{\prime}\right)=\iint d p d q f_{\mathrm{DGCM}}(q, p) \Gamma\left(q, p ; q^{\prime}\right) .
$$

Here, $f_{\mathrm{GCM}}(q)$ and $f_{\mathrm{DGCM}}(q, p)$ are the weight functions in Eqs. (1) and (7), respectively. In this case, there is no need to consider the DGCM and the usual GCM is sufficient. This condition is called the global redundancy [39]. Goeke and Reinhard further showed that there are certain cases in which a one-parameter GCM along a path in the $(q, p)$ space suffices even without the global redundancy [39]. However, in general, the configuration along such a relevant path is considerably complicated. In that situation, one can instead apply the DGCM in a straightforward manner. Nevertheless, it is not numerically easy to construct the configurations along a dynamical path, partly because the number of collective variables is doubled in the DGCM. For this reason, the DGCM has not yet been applied to any concrete numerical problems.

\section{B. Dynamical path from a constrained Hartree-Fock method}

Another potential problem of the DGCM is that it is not obvious how to practically find a dynamical path. In this regard, we show below that there is a reasonable way to construct a dynamical path when a collective coordinate is generated by the constrained Hartree-Fock(-Bogoliubov) method.

Suppose that $|q\rangle$ is a many-body state which satisfies

$$
\left\langle q\left|\hat{Q}_{0}\right| q\right\rangle=q
$$


with a Hermitian operator $\hat{Q}_{0}$. We then define a state

$$
|q, p\rangle \equiv e^{i \hat{Q}_{0} p}|q\rangle .
$$

It is obvious that this state satisfies

$$
\left\langle q, p\left|\hat{Q}_{0}\right| q, p\right\rangle=q .
$$

Differentiating both sides of this equation by $q$, we then obtain

$$
\left\langle q, p\left|\overleftarrow{\partial}_{q} \hat{Q}_{0}+\hat{Q}_{0} \vec{\partial}_{q}\right| q, p\right\rangle=1
$$

Noticing

$$
i \hat{Q}_{0}|q, p\rangle=\vec{\partial}_{p}|q, p\rangle,
$$

which follows from the definition of the state $|q, p\rangle$, Eq. (11), we find

$$
\left\langle q, p\left|\overleftarrow{\partial}_{q} \vec{\partial}_{p}-\overleftarrow{\partial}_{p} \vec{\partial}_{q}\right| q, p\right\rangle=i
$$

This is nothing more than the conjugate condition, Eq. (3). That is, when one uses the constrained Hartree-Fock(Bogoliubov) method to generate a collective coordinate with a Hermitian operator, one can always construct a desired dynamical path.

If one employs the states $|q, p\rangle$ so obtained in the DGCM, a many-body wave function is expressed as

$$
|\psi\rangle=\iint d q d p f(q, p) e^{i \hat{Q}_{0} p}|q\rangle .
$$

We rewrite this equation using the Fourier transform of $f(q, p)$,

$$
f(q, p)=\int d q^{\prime} \tilde{f}\left(q, q^{\prime}\right) e^{-i q^{\prime} p} .
$$

This leads to

$$
|\psi\rangle=\iint d q d q^{\prime} \tilde{f}\left(q, q^{\prime}\right)\left\{\int d p e^{i\left(\hat{Q}_{0}-q^{\prime}\right) p}\right\}|q\rangle .
$$

Notice that, apart from the normalization coefficient,

$$
\hat{P}_{q^{\prime}}^{\left(\hat{Q}_{0}\right)} \equiv \int d p e^{i\left(\hat{Q}_{0}-q^{\prime}\right) p}
$$

is the projection operator which projects a state onto an eigenfunction of the operator $\hat{Q}_{0}$ with an eigenvalue of $q^{\prime}$. Thus, the DGCM state can be expressed as

$$
|\psi\rangle=\iint d q d q^{\prime} \tilde{f}\left(q, q^{\prime}\right) \hat{P}_{q^{\prime}}^{\left(\hat{Q}_{0}\right)}|q\rangle
$$

This implies that the DGCM is equivalent to the GCM supplemented by a projection method.

\section{Translational motion}

Let us apply the formula derived in the previous subsection to the center of mass motion and compare with the double projection method. To this end, we first generate the state $|\boldsymbol{p}\rangle$ which satisfies

$$
\langle\boldsymbol{p}|\hat{\boldsymbol{P}}| \boldsymbol{p}\rangle=\boldsymbol{p},
$$

where $\hat{\boldsymbol{P}}=\left(\hat{P}_{x}, \hat{P}_{y}, \hat{P}_{z}\right)$ is the operators for the center of mass motion of a whole system. Since $\hat{P}_{x}, \hat{P}_{y}$, and $\hat{P}_{z}$ commute with each other, the wave function in the DGCM, Eq. (18), reads

$$
|\psi\rangle=\iint d \boldsymbol{p}^{\prime} d \boldsymbol{p}^{\prime \prime} \tilde{f}\left(\boldsymbol{p}^{\prime}, \boldsymbol{p}^{\prime \prime}\right)\left\{\int d \boldsymbol{q} e^{-i\left(\hat{\boldsymbol{P}}-\boldsymbol{p}^{\prime \prime}\right) \cdot \boldsymbol{q}}\right\}\left|\boldsymbol{p}^{\prime}\right\rangle .
$$

Since the operators $\hat{\boldsymbol{P}}$ commutes with the Hamiltonian, one would be interested only in the eigenstates of $\hat{\boldsymbol{P}}$. Acting the projection operator for the operator $\hat{\boldsymbol{P}}$ onto Eq. (22), one then obtains

$$
|\psi\rangle_{\boldsymbol{p}}=\iint d \boldsymbol{q} d \boldsymbol{p}^{\prime} \tilde{f}\left(\boldsymbol{p}^{\prime}, \boldsymbol{p}\right) e^{-i(\hat{\boldsymbol{P}}-\boldsymbol{p}) \cdot \boldsymbol{q}}\left|\boldsymbol{p}^{\prime}\right\rangle .
$$

This coincides with Eq. (2.6) in Ref. [27]. In this way, the ansatz of the double projection method can be directly derived from the DGCM, when the operators to generate a generator coordinate commutes with a Hamiltonian. In this sense, the DGCM can be regarded as an extension of the double projection method of Peierls and Thouless [27].

Incidentally, we mention that another approach with the variation after projection (VAP) method for the translational motion has also been carried out in Refs. [10,11].

\section{Rotational motion}

Let us next consider a rotational motion, thus, the angular momentum. For the sake of simplicity, we consider only a rotation around the $x$ axis. Using the operator $\hat{J}_{x}$, we first generate a generator coordinate imposing a condition of

$$
\left\langle m\left|\hat{J}_{x}\right| m\right\rangle=m \text {. }
$$

Following the same procedure as in the center of mass motion, one can write the DGCM ansatz for the eigenstates of $\hat{J}_{x}$ as

$$
|\psi\rangle_{m}=\iint d \theta d m^{\prime} f\left(m^{\prime}, m\right) e^{-i\left(\hat{J}_{x}-m\right) \theta}\left|m^{\prime}\right\rangle .
$$

This is also consistent with Eq. (3.11) in Ref. [27].

Unfortunately, it is not straightforward to extend this discussion to a general rotation, since the angular momentum operators $\hat{J}_{x}, \hat{J}_{y}$, and $\hat{J}_{z}$ do not commute with each other, unlike the linear momentum operators, $\hat{\boldsymbol{P}}$. One possible prescription is to construct a DGCM wave function using $\hat{J}_{x}$ and $\hat{\boldsymbol{J}}^{2}$. Wave functions similar to this have been considered in Refs. [28-32], in which the following ansatz was employed:

$$
|L M\rangle=\sum_{K, m^{\prime}} f_{K m^{\prime}} \hat{P}_{M K}^{L}\left|m^{\prime}\right\rangle .
$$

Here, $m^{\prime}$ is the generator coordinate defined by Eq. (24) and $\hat{P}_{M K}^{L}$ is the angular momentum projection operator. We have dropped other parameters than the angular momentum, such as deformation, from the notation in Eq. (26). Notice that the idea of DGCM is applied in this equation only to the angular momentum component in the direction of the quantization axis, while the effect of the fluctuation of the total angular momentum is not considered. It might be an interesting future work to extend this prescription by introducing a generator coordinate associated with the total angular momentum in addition to that in Eq. (24). 


\section{E. Particle number}

We next consider the particle number projection. Using the particle number operator $\hat{N}$, we first generate a generator coordinate according to

$$
\langle N|\hat{N}| N\rangle=N .
$$

Here, the state $|N\rangle$ represents either a BCS state or a HartreeFock-Bogoliubov state, in which several particle number components are mixed. If we construct the eigenstate of $\hat{N}$, one can write the DGCM wave function as

$$
|\psi\rangle_{N_{0}}=\iint d N d \phi f\left(N, N_{0}\right) e^{i\left(\hat{N}-N_{0}\right) \phi}|N\rangle .
$$

Here, $\phi$ is the gauge angle, which is a quantity conjugate to the particle number. If one considers a nonrelativistic case, the particle number operator is semipositive definite. In this case, the integral range of $N$ is from 0 to $\infty$. On the other hand, for $\phi$, the range of the integral is from 0 to $2 \pi$. Using the particle number projection operator defined by

$$
\hat{P}^{N_{0}}=\int_{0}^{2 \pi} \frac{d \phi}{2 \pi} e^{i\left(\hat{N}-N_{0}\right) \phi},
$$

one thus has

$$
|\psi\rangle_{N_{0}}=\int_{0}^{\infty} d N f_{N_{0}}(N) \hat{P}^{N_{0}}|N\rangle,
$$

except for a normalization constant. This can also be interpreted as the double projection method for the particle number fluctuation. The Hill-Wheeler equation for $f_{N_{0}}(N)$ reads

$$
\int_{0}^{\infty} d N^{\prime}\left(\left\langle N\left|\hat{H} \hat{P}^{N_{0}}\right| N^{\prime}\right\rangle-E\left\langle N\left|\hat{P}^{N_{0}}\right| N^{\prime}\right\rangle\right) f_{N_{0}}\left(N^{\prime}\right)=0 .
$$

Notice that, in the case of the particle number, the usual GCM corresponds to the variation before projection (VBP) method,

$$
|\psi\rangle_{N} \propto \int_{0}^{2 \pi} \frac{d \phi}{2 \pi} e^{i(\hat{N}-N) \phi}|N\rangle .
$$

In addition to the gauge angle $\phi$, if one treats $N$ as a generator coordinate, one obtains the DGCM wave function, Eq. (30). While the VBP takes into account only the fluctuation of the gauge angle, the DGCM incorporates the effect of the fluctuation of a mean particle number in mean-field wave functions.

Even though the DGCM, or the double projection method, has not been applied to the particle number fluctuation, GCM calculations based on a similar idea have been carried out treating the pairing fluctuation as a generator coordinate [40-42]. See also Ref. [43]. There, the generating functions are constructed as

$$
\left|\left\langle\delta\left|(\Delta \hat{N})^{2}\right| \delta\right\rangle\right|^{1 / 2}=\delta,
$$

using the operator $\Delta \hat{N}=\hat{N}-\langle\hat{N}\rangle$. Following the idea of $\mathrm{GCM}$, these wave functions are linearly superposed as

$$
|\psi\rangle_{N_{0}}=\int d \delta f(\delta) \hat{P}^{N_{0}}|\delta\rangle .
$$

It has been shown that such treatment of the pair fluctuation improves the description of the structure of ${ }^{54} \mathrm{Cr}$ [40] and also significantly affects nuclear matrix elements of double beta decays [42]. This method indeed takes into account the effect of pairing fluctuation, but it is not clear whether it fully takes it into account in a sense of the DGCM illustrated in this subsection.

\section{NUMERICAL CALCULATIONS FOR DGCM FOR PARTICLE NUMBER}

\section{A. Numerical details}

In this section, we apply the DGCM to actual nuclei and numerically investigate the effect of the particle number fluctuation in a BCS wave function using Eq. (30). To this end, we focus for simplicity only on the neuron number. We thus choose the singly closed ${ }^{16,18} \mathrm{O},{ }^{40,42} \mathrm{Ca}$, and ${ }^{56,58,64} \mathrm{Ni}$ nuclei and assume that the protons are in the normal fluid phase. Considering the systematic calculation [44], we also assume that these nuclei have a spherical symmetry. We employ the SIII Skyrme energy functional [45].

We prepare a set of many-body wave functions $|N\rangle$ which have the average particle number of $N$. Notice that $N$ may be different from the actual neutron number $N_{0}$ for each nucleus, and that $N$ may not necessarily be an integer number. For this purpose, we employ the BCS approximation for the pairing correlation among neutrons, while we ignore the neutronproton pairing. We solve the Skyrme-Hartree-Fock equation in a box with $15 \mathrm{fm}$ with a mesh spacing of $0.05 \mathrm{fm}$, and the continuum states are then discretized. To calculate the pairing energy, we employ the pairing energy functional given by

$$
E_{\text {pair }}[\rho, \tilde{\rho}]=\frac{V_{n}}{4} \int d \boldsymbol{r}\left(1-\frac{\rho(\boldsymbol{r})}{\rho_{0}}\right)\left|\tilde{\rho}_{n}(\boldsymbol{r})\right|^{2},
$$

where $\rho(\boldsymbol{r})$ and $\tilde{\rho}_{n}(\boldsymbol{r})$ are the total particle density and the neutron pair density, respectively, with $\rho_{0}$ being $0.16 \mathrm{fm}^{-3}$. The pairing energy is calculated with an energy cut-off at 15 $\mathrm{MeV}$ above the Fermi energy.

For ${ }^{18} \mathrm{O},{ }^{42} \mathrm{Ca}$, and ${ }^{58,64} \mathrm{Ni}$, we determine the value of $V_{n}$ so that the average pairing gap,

$$
\bar{\Delta}=\frac{\frac{V_{n}}{2} \int d^{3} r\left(1-\frac{\rho(\boldsymbol{r})}{\rho_{0}}\right)\left|\tilde{\rho}_{n}(\boldsymbol{r})\right|^{2}}{\int d^{3} r\left|\tilde{\rho}_{n}(\boldsymbol{r})\right|^{2}},
$$

coincides with the empirical value, $12 / \sqrt{A} \mathrm{MeV}$, where $A$ is the mass number of a nucleus. For the doubly magic nuclei, ${ }^{16} \mathrm{O}$ and ${ }^{40} \mathrm{Ca}$, we somewhat reduce the value of $V_{n}$ so that the pairing gap becomes zero in the BCS approximation. For the ${ }^{56} \mathrm{Ni}$ nucleus, this problem does not appear and we use the same value of $V_{n}$ as that for ${ }^{58} \mathrm{Ni}$. The parameters are listed in Table I.

We mainly show below the results with $\Delta N=0.2$ around $N_{0}$ in the range of $N_{0}-2 \leqslant N \leqslant N_{0}+2$. For simplicity, for each nucleus we ignore the nonorthogonality of singleparticle wave functions for different values of $N$ and assume $\left\langle\varphi_{i}(N) \mid \varphi_{j}\left(N^{\prime}\right)\right\rangle=\delta_{i, j}$, where $\varphi_{i}(N)$ is the $i$ th single-particle wave function for a system with the average neutron number of $N$. We have confirmed that the deviation from this condition is negligibly small in the range of $N$ considered in this paper. We thus use the single-particle wave functions for $N=N_{0}$ for each nucleus. 
TABLE I. The strengths of the pairing interaction, $V_{n}$, employed in the present calculations. These are given in units of $\mathrm{MeV}_{\mathrm{fm}}{ }^{-3}$.

\begin{tabular}{lcccccc}
\hline \hline nucleus & ${ }^{16} \mathrm{O}$ & ${ }^{18} \mathrm{O}$ & ${ }^{40} \mathrm{Ca}$ & ${ }^{42} \mathrm{Ca}$ & ${ }^{56} \mathrm{Ni}$ & ${ }^{58} \mathrm{Ni}$ \\
\hline$V_{n}$ & -800.00 & -901.98 & -700.00 & -775.23 & -897.80 & -897.80 \\
\hline \hline
\end{tabular}

Figure 1 shows the probability of the component of $N_{0}$ in each BCS wave function $|N\rangle$ as a function of $N$ for the ${ }^{56} \mathrm{Ni}$ nucleus $\left(N_{0}=28\right)$. This is computed as $P_{N}^{N_{0}}=\left\langle N\left|\hat{P}^{N_{0}}\right| N\right\rangle$, with the particle number projection operator, Eq. (29). One can see that the probability has a large value in the range considered in this paper, $N_{0}-2 \leqslant N \leqslant N_{0}+2$. The BCS states with larger values of $N$ have a smaller overlap with the state with $N_{0}$, and inclusion of such states in the DGCM may cause a serious numerical problem.

We then apply the particle number projection, Eq. (29), to the wave functions $|N\rangle$ and superpose them according to Eq. (30). For this purpose, we discretize the gauge angle $\phi$ with $\Delta \phi=2 \pi / 80$ for the integral with respect to $\phi$. We use the mixed density prescription to calculate the Hamiltonian and the overlap kernels $[46,47]$. Since we use the same singleparticle wave functions for each $N$, the mixed density and the mixed pair density are simply given by

$$
\begin{aligned}
& \rho_{N N^{\prime}}^{\phi}(\boldsymbol{r})=\sum_{i} \frac{v_{i}^{N} v_{i}^{N^{\prime}} e^{2 i \phi}}{u_{i}^{N} u_{i}^{N^{\prime}}+v_{i}^{N} v_{i}^{N^{\prime}} e^{2 i \phi}}\left|\varphi_{i}(\boldsymbol{r})\right|^{2}, \\
& \tilde{\rho}_{N N^{\prime}}^{\phi}(\boldsymbol{r})=\sum_{i} \frac{u_{i}^{N} v_{i}^{N^{\prime}} e^{2 i \phi}}{u_{i}^{N} u_{i}^{N^{\prime}}+v_{i}^{N} v_{i}^{N^{\prime}} e^{2 i \phi}}\left|\varphi_{i}(\boldsymbol{r})\right|^{2},
\end{aligned}
$$

respectively. Here, $u_{i}^{N}$ and $v_{i}^{N}$ are the $u v$ factors for the singleparticle state $i$ in the BCS wave function with the average neutron number $N$. Other local mixed densities are given in a similar way.

In our calculations, we superpose many similar states. The problem of overcompleteness may then arise [26] due to the linear dependence of the bases. To avoid this problem, in numerical calculations shown below, we remove the eigenstates of the overlap kernel whose eigenvalue is smaller than $\lambda_{\text {cut }}=$

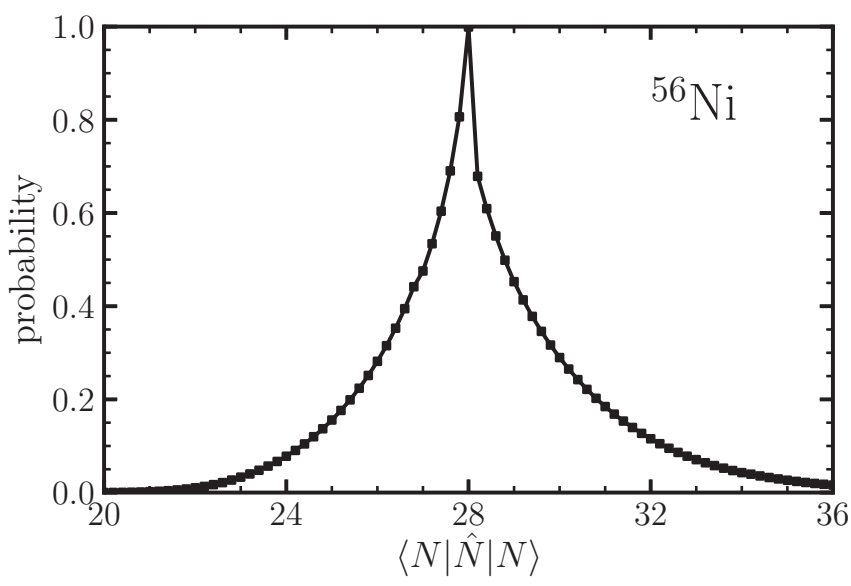

FIG. 1. The probability to find the $N_{0}=28$ component in the BCS wave function $|N\rangle$ for ${ }^{56} \mathrm{Ni}$ which has the average neutron number of $N$.
$10^{-5}$ (see Fig. 2 below for the dependence of the result on the choice of $\left.\lambda_{\text {cut }}\right)$. In the actual calculations, with this remedy for the overcompleteness, we use the subroutine DSYEV of the LAPACK package [48] to diagonalize the discretized HillWheeler equation as both the Hamiltonian and the overlap kernels are real symmetric matrices in the present calculation.

\section{B. Results}

Figure 2 shows the total energy gain $\Delta E$ for ${ }^{56} \mathrm{Ni}$ due to the superposition of various $|N\rangle$ states in Eq. (30). Here, the energy gain is defined as $\Delta E=E\left(N_{\mathrm{DGCM}}\right)-E\left(N_{\mathrm{DGCM}}=1\right)$, where $E\left(N_{\mathrm{DGCM}}\right)$ is the total energy of the system when the number of basis is $N_{\text {DGCM }}$. This quantity is plotted as a function of the number of basis $(|N\rangle), N_{\text {DGCM }}$, where $N_{\text {DGCM }}=1$ corresponds to the usual variation before particle number projection (VBP). To draw the figure, we increase the number of basis by adding two basis states symmetrically around $N_{0}$, that is, $N_{0}, N_{0} \pm \Delta N, N_{0} \pm 2 \Delta N \ldots$ The solid, the dashed, and the dotted lines denote the results with $\lambda_{\text {cut }}=10^{-4}, 10^{-5}$, and $10^{-6}$ for the cut-off of the eigenvalues of the overlap kernel, respectively. One can see that the results are almost converged at $\lambda_{\text {cut }}=10^{-5}$. We thus use this value in all the calculations shown below unless otherwise mentioned. The figure also shows that the energy gain quickly converges as a function of $N_{\text {DGCM }}$. In particular, the energy is significantly decreased even with a mixture of three basis states only, $N_{\mathrm{DGCM}}=3$. We have repeated the same calculation with $\Delta N=0.1$ and have found that the converged energy remains almost the same

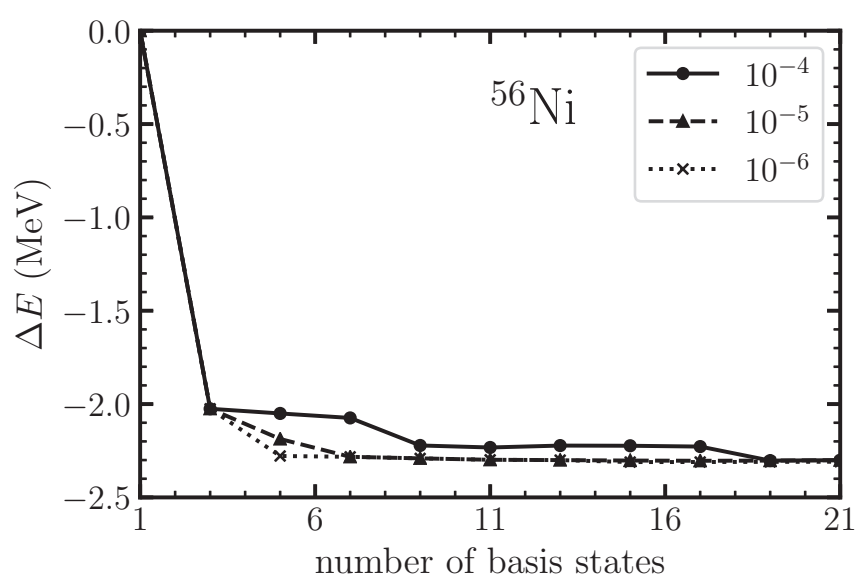

FIG. 2. The energy gain in the ${ }^{56} \mathrm{Ni}$ nucleus as a function of the number of basis states $N_{\mathrm{DGCM}}$ in the DGCM method. It is plotted with respect to the energy of $N_{\mathrm{DGCM}}=1$, which is equivalent to the variation before projection method. The solid, the dashed, and the dotted lines denote the results with $\lambda_{\text {cut }}=10^{-4}, 10^{-5}$, and $10^{-6}$ for the cut-off of the eigenvalues of the overlap kernel, respectively. 
TABLE II. The ground-state energy of each nucleus calculated with different methods. The energies are given in units of $\mathrm{MeV}$. For the DGCM method, the number in the parenthesis denotes the number of basis states, $N_{\mathrm{GCM}}$, for which DGCM(1) is equivalent to VBP.

\begin{tabular}{lcccc}
\hline \hline & BCS & DGCM(1) & DGCM(3) & DGCM(21) \\
\hline${ }^{16} \mathrm{O}$ & -128.01 & -128.01 & -128.41 & -129.29 \\
${ }^{18} \mathrm{O}$ & -144.91 & -147.50 & -147.82 & -148.03 \\
${ }^{40} \mathrm{Ca}$ & -341.30 & -341.30 & -342.63 & -342.79 \\
${ }^{42} \mathrm{Ca}$ & -363.83 & -365.55 & -365.87 & -365.98 \\
${ }^{56} \mathrm{Ni}$ & -482.74 & -482.74 & -484.76 & -485.04 \\
${ }^{58} \mathrm{Ni}$ & -504.40 & -506.31 & -507.37 & -507.79 \\
${ }^{64} \mathrm{Ni}$ & -557.87 & -559.44 & -559.66 & -559.84 \\
\hline \hline
\end{tabular}

as that with $\Delta N=0.2$, with a similar convergence feature to each other.

Table II summarizes the results for the ${ }^{16,18} \mathrm{O},{ }^{40,42} \mathrm{Ca}$, and $56,58,64 \mathrm{Ni}$ nuclei. ${ }^{1}$ One can see that a large energy gain is obtained for all of these cases, as in ${ }^{56} \mathrm{Ni}$ shown in Fig. 2. As we have discussed in Sec. II D, this can be interpreted as a consequence of the fluctuation of a mean particle number in mean-field wave functions. It is noteworthy that the energy gain is particularly large for the neutron magic nuclei, ${ }^{16} \mathrm{O}$, ${ }^{40} \mathrm{Ca}$, and ${ }^{56} \mathrm{Ni}$. To clarify the reason for this, we show in Fig. 3 the energy gain (the solid lines) and the contribution of the pairing energy (the dashed lines) as a function of $N_{\text {DGCM }}$. The lines with the filled circles denote the results for ${ }^{56} \mathrm{Ni}$, while the lines with the filled triangles are for ${ }^{58} \mathrm{Ni}$. One can clearly

${ }^{1}$ For ${ }^{16} \mathrm{O}$, the problem of overcompleteness is found to be severe, and we chose $\lambda_{\text {cut }}=8.0 \times 10^{-2}$, which is determined from the eigenvalue distribution of the overlap kernel.

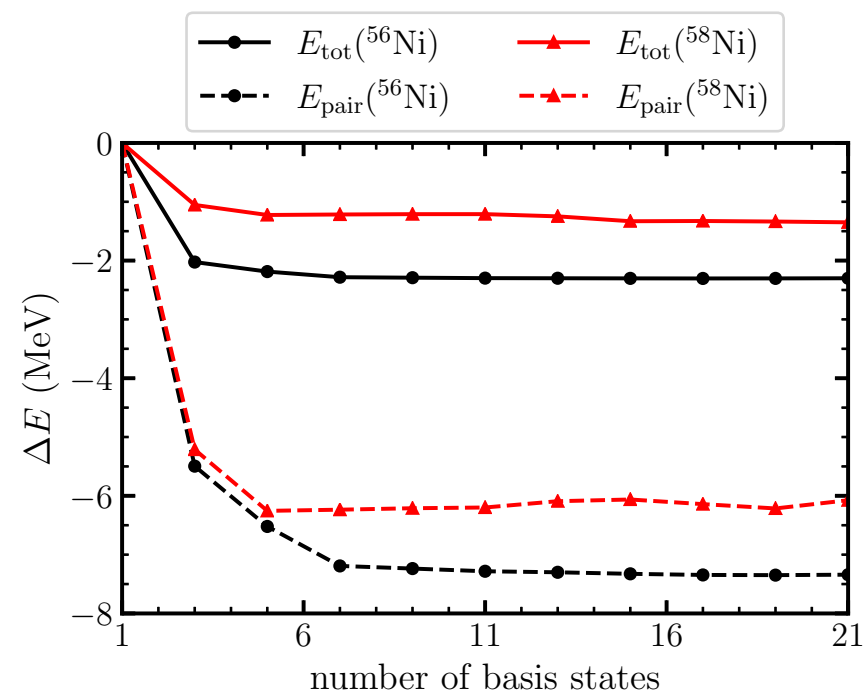

FIG. 3. Similar to Fig. 2, but for a comparison between the total energy (the solid lines) and the pairing energy (the dashed lines). The filled circles and the filled triangles denote the results for the ${ }^{56} \mathrm{Ni}$ and the ${ }^{58} \mathrm{Ni}$ nuclei, respectively.

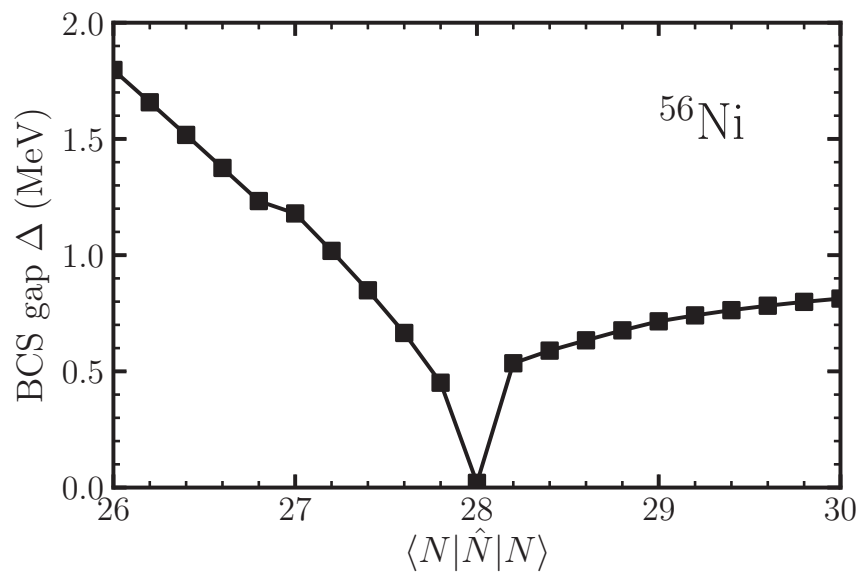

FIG. 4. The pairing gap $\Delta$ of ${ }^{56} \mathrm{Ni}$ in the BCS approximation for the basis states for the DGCM calculation.

see that the total energy decreases with the development of the pairing energy. It is interesting to notice that the pairing contribution is larger in the neutron magic nucleus ${ }^{56} \mathrm{Ni}$ as compared to that in ${ }^{58} \mathrm{Ni}$. This is due to the fact that, for ${ }^{58} \mathrm{Ni}$, the effect of the pairing correlation is already taken into account to some extent in the calculation with $N_{\mathrm{DGCM}}=1$, while for ${ }^{56} \mathrm{Ni}$ the energy with VBP does not change from that in the BCS approximation due to the absence of the pairing gap. To illustrate this, Fig. 4 shows the BCS pairing gap for the basis states $|N\rangle$ for ${ }^{56} \mathrm{Ni}$ used in this study. While the pairing gap is zero for $N=28$, the gap is finite for other basis states. Therefore, this nucleus can take an advantage of finite pairing gaps by mixing configurations with $\langle\hat{N}\rangle \neq 28$, which significantly lowers the total energy. In this sense, the DGCM for the particle number is somewhat similar to the GCM calculations where a pairing fluctuation is treated as a generator coordinate [41,49-51]. It is also noted that the energy gain due to DGCM is small for nuclei where the pairing correlation is well developed, such as ${ }^{64} \mathrm{Ni}$ shown in Table II.

In the mean-field calculations, the VAP method is more consistent than the VBP method [26,52-57]. However, the VAP is much more cumbersome and is often numerically more involved as compared to the VBP. One may resort to the Lipkin-Nogami method (LN) [58,59] as an approximation of the VAP, but it has been know that the LN method does not work well for nuclei closed to shell closures [60-63]. The method proposed in this paper is much simpler than the VAP, yet a similar amount of the energy gain can be obtained with a lower computation cost. In particular, it is a numerical advantage of our method that the total energy is significantly lowered already with $N_{\text {DGCM }}=3$. Moreover, our method works well not only for open shell nuclei but also for nuclei close to a shell closure. We thus argue that our method can be a good alternative to the VAP and the Lipkin-Nogami methods.

\section{SUMMARY AND FUTURE PERSPECTIVES}

We have discussed an extension of the generator coordinate method (GCM) by treating both a collective coordinate and 
its conjugate momentum as generator coordinates. To this end, we have investigated the idea of the dynamical GCM (DGCM). We have first shown that a dynamical path relevant to the DGCM can be constructed whenever a collective coordinate is generated by the constrained mean-field method with a Hermitian operator. The DGCM can thus be applied once the operator relevant to a collective motion is identified. In such cases, the DGCM can be formulated in a form of a generalized double projection method.

We have applied the DGCM to the particle number projection as an example. Here, we have superposed many BCS states which have different mean particle numbers, after performing the particle number projection. In this way, we have incorporated not only the fluctuation of the gauge angle but also the fluctuation of the mean particle number in the BCS wave functions. As a result, the ground state energy was found to be significantly lowered compared to the BCS + VBP method, which can be regarded as the usual GCM for the gauge angle. In particular, for magic nuclei, no energy gain is obtained in the VBP method due to a vanishing of pairing correlation in the mean field approximation, while this method yields a significant energy gain by mixing configurations with a nonzero pairing gap. This consideration may be important for the Mottelson-Valatin effect [64] in nuclear superconductivity at high angular momenta and/or at high temperatures. There, the fluctuation beyond the mean field approximation may play an important role $[65,66]$, and it may be interesting to recast this problem from the viewpoint of DGCM.

We have also found that the convergence of the DGCM calculations is fast with respect to the number of superposed states, and a considerable energy gain is obtained by mixing only three configurations. The method is much simpler than the VAP, and thus a numerical calculation is much easier. Moreover, the method works well for both open shell nuclei and magic nuclei, unlike the Lipkin-Nogami method which does not work for nuclei at shell closures. We thus advocate this method as a good alternative to the VAP method.

In this paper, for simplicity we have carried out all the calculations assuming spherical symmetry. It would be an interesting future work to extend this by removing the restriction of nuclear shape. This would be important particularly for soft nuclei, for which the nuclear shape may change significantly as a function of the average particle number. In such cases, it is an advantage of our method that correlations associated with the shape degree of freedom can be largely incorporated by mixing a few configurations with different average particle numbers.

In addition, even though we have focused in this paper only on the neutron number, it is straightforward to include the fluctuation of the average proton number in BCS states as well. The resultant DGCM wave function can easily incorporate the effect of a proton-neutron pairing, because it is no longer a form of the direct product of proton and neutron states. This may be a good advantage to explaining the Wigner energy $[67,68]$, for which the fluctuation of a pair field may play an important role [69,70].

Our calculations presented in this paper indicates that a better description can be achieved with the DGCM by incorporating the conjugate momentum of a collective coordinate. This would mean that GCM calculations reported in the literature may need to be reexamined from the view point of the DGCM. For example, for a GCM calculation with the quadrupole deformation operator $\hat{Q}_{20}$, the DGCM wave function can be constructed according to Eq. (16) or (20). This would require a development of a computation method to evaluate the operator in a form of $\exp \left(i q \hat{Q}_{20}\right)$ or to carry out the quantum number projection for the operator $\hat{Q}_{20}$. Since the multipole operators are mutually commutative, the DGCM wave function can be easily extended to a multi-dimensional deformation plane as well. It would be an interesting future problem to develop a microscopic fission theory [71] based on this idea.

\section{ACKNOWLEDGMENTS}

The authors thank N. Hinohara, J. L. Egido, and K. Pomorski for useful discussions. This work was supported by JSPS KAKENHI (Grants No. JP19K03824, No. JP19K03861, and No. JP19K03872).
[1] M. Bender, P.-H. Heenen, and P.-G. Reinhard, Rev. Mod. Phys. 75, 121 (2003).

[2] T. Nikšić, D. Vretenar, and P. Ring, Prog. Part. Nucl. Phys. 66, 519 (2011).

[3] J. L. Egido, Phys. Scr. 91, 073003 (2016).

[4] L. M. Robledo, T. R. Rodríguez, and R. R. Rodríguez-Guzmán, J. Phys. G: Nucl. Part. 46, 013001 (2018).

[5] M. Bender and P.-H. Heenen, Nucl. Phys. A 713, 390 (2003).

[6] T. Duguet, M. Bender, P. Bonche, and P.-H. Heenen, Phys. Lett. B 559, 201 (2003).

[7] M. Bender, H. Flocard, and P. H. Heenen, Phys. Rev. C 68 , 044321 (2003).

[8] R. R. Rodríguez-Guzmán, J. L. Egido, and L. M. Robledo, Phys. Rev. C 69, 054319 (2004).

[9] M. Bender, P.-H. Heenen, and P. Bonche, Phys. Rev. C 70, 054304 (2004).
[10] R. R. Rodríguez-Guzmán and K. W. Schmid, Eur. Phys. J. A 19, 45 (2004).

[11] R. R. Rodríguez-Guzmán and K. W. Schmid, Eur. Phys. J. A 19, 61 (2004).

[12] S. Shinohara, H. Ohta, T. Nakatsukasa, and K. Yabana, Phys. Rev. C 74, 054315 (2006).

[13] T. R. Rodríguez and J. L. Egido, Phys. Rev. Lett. 99, 062501 (2007).

[14] T. R. Rodríguez and J. L. Egido, Phys. Lett. B 663, 49 (2008).

[15] M. Bender and P.-H. Heenen, Phys. Rev. C 78, 024309 (2008).

[16] J. M. Yao, J. Meng, P. Ring, and D. Vretenar, Phys. Rev. C 81, 044311 (2010).

[17] T. R. Rodríguez and J. L. Egido, Phys. Rev. C 81, 064323 (2010).

[18] T. R. Rodríguez and J. L. Egido, Phys. Rev. C 84, 051307(R) (2011). 
[19] T. R. Rodríguez and J. L. Egido, Phys. Lett. B 705, 255 (2011).

[20] J. M. Yao, M. Bender, and P.-H. Heenen, Phys. Rev. C 87, 034322 (2013).

[21] Y. Fukuoka, S. Shinohara, Y. Funaki, T. Nakatsukasa, and K. Yabana, Phys. Rev. C 88, 014321 (2013).

[22] B. Bally, B. Avez, M. Bender, and P.-H. Heenen, Phys. Rev. Lett. 113, 162501 (2014).

[23] J. M. Yao, K. Hagino, Z. P. Li, J. Meng, and P. Ring, Phys. Rev. C 89, 054306 (2014).

[24] J. M. Yao, E. F. Zhou, and Z. P. Li, Phys. Rev. C 92, 041304(R) (2015).

[25] J. L. Egido and A. Jungclaus, Phys. Rev. Lett. 125, 192504 (2020).

[26] P. Ring and P. Schuck, The Nuclear Many-Body Problem (Springer-Verlag, New York, 1980).

[27] P. Peierls and D. Thouless, Nucl. Phys. 38, 154 (1962).

[28] M. Borrajo, T. R. Rodríguez, and J. L. Egido, Phys. Lett. B 746, 341 (2015).

[29] J. L. Egido, M. Borrajo, and T. R. Rodríguez, Phys. Rev. Lett. 116, 052502 (2016).

[30] M. Shimada, S. Tagami, and Y. R. Shimizu, Prog. Theo. Exp. Phys 2015, $063 \mathrm{D} 02$ (2015).

[31] M. Shimada, S. Tagami, and Y. R. Shimizu, Phys. Rev. C 93, 044317 (2016).

[32] M. Ushitani, S. Tagami, and Y. R. Shimizu, Phys. Rev. C 99, 064328 (2019).

[33] B. Jancovici and D. Schiff, Nucl. Phys. 58, 678 (1964).

[34] D. Brink and A. Weiguny, Nucl. Phys. A 120, 59 (1968).

[35] K. Goeke and P.-G. Reinhard, Ann. Phys. (NY) 112, 328 (1978).

[36] P. G. Reinhard and K. Goeke, Phys. Rev. C 17, 1249 (1978).

[37] P. G. Reinhard and K. Goeke, J. Phys. G 4, L245 (1978).

[38] P.-G. Reinhard and K. Goeke, Nucl. Phys. A 312, 121 (1978).

[39] K. Goeke and P.-G. Reinhard, Ann. Phys. (NY) 124, 249 (1980).

[40] N. López Vaquero, T. R. Rodriguez, and J. L. Egido, Phys. Lett. B 704, 520 (2011).

[41] R. A. Broglia and V. Zelevinsky, Fifty Years of Nuclear BCS (World Scientific, Singapore, 2013).

[42] N. L. Vaquero, T. R. Rodríguez, and J. L. Egido, Phys. Rev. Lett. 111, 142501 (2013).

[43] S. A. Giuliani, L. M. Robledo, and R. Rodríguez-Guzmán, Phys. Rev. C 90, 054311 (2014).

[44] J. Erler, N. Birge, M. Kortelainen, W. Nazarewicz, E. Olsen, A. M. Perhac, and M. Stoitsov, Nature 486, 509 (2012).

[45] M. Beiner, H. Flocard, N. Van Giai, and P. Quentin, Nucl. Phys. A 238, 29 (1975).
[46] J. Dobaczewski, M. V. Stoitsov, W. Nazarewicz, and P.-G. Reinhard, Phys. Rev. C 76, 054315 (2007).

[47] P. Bonche, J. Dobaczewski, H. Flocard, P.-H. Heenen, and J. Meyer, Nucl. Phys. A 510, 466 (1990).

[48] LAPACK, http://www.netlib.org/lapack/.

[49] A. Góźdź, K. Pomorski, M. Brack, and E. Werner, Nucl. Phys. A 442, 50 (1985).

[50] M. A. Fernández and J. L. Egido, Phys. Rev. B 68, 184505 (2003).

[51] M. A. Fernández and J. L. Egido, Eur. Phys. J. B 48, 305 (2005).

[52] J. Sheikh and P. Ring, Nucl. Phys. A 665, 71 (2000).

[53] J. A. Sheikh, P. Ring, E. Lopes, and R. Rossignoli, Phys. Rev. C 66, 044318 (2002).

[54] M. V. Stoitsov, J. Dobaczewski, R. Kirchner, W. Nazarewicz, and J. Terasaki, Phys. Rev. C 76, 014308 (2007).

[55] T. Duguet, M. Bender, K. Bennaceur, D. Lacroix, and T. Lesinski, Phys. Rev. C 79, 044320 (2009).

[56] G. Hupin and D. Lacroix, Phys. Rev. C 86, 024309 (2012).

[57] B. Bally and M. Bender, Phys. Rev. C 103, 024315 (2021).

[58] H. J. Lipkin, Ann. Phys. (NY) 9, 272 (1960).

[59] Y. Nogami, Phys. Rev. 134, B313 (1964).

[60] D. C. Zheng, D. W. L. Sprung, and H. Flocard, Phys. Rev. C 46, 1355 (1992).

[61] J. Dobaczewski and W. Nazarewicz, Phys. Rev. C 47, 2418 (1993).

[62] K. Hagino and G. Bertsch, Nucl. Phys. A 679, 163 (2000).

[63] K. Hagino, P.-G. Reinhard, and G. F. Bertsch, Phys. Rev. C 65, 064320 (2002).

[64] B. R. Mottelson and J. G. Valatin, Phys. Rev. Lett. 5, 511 (1960).

[65] J. Egido, P. Ring, S. Iwasaki, and H. Mang, Phys. Lett. B 154, 1 (1985).

[66] Y. Shimizu and R. Broglia, Nucl. Phys. A 515, 38 (1990).

[67] E. Wigner, Phys. Rev. 51, 106 (1937).

[68] P. Van Isacker, D. D. Warner, and D. S. Brenner, Phys. Rev. Lett. 74, 4607 (1995).

[69] W. Satuła and R. Wyss, Phys. Lett. B 393, 1 (1997).

[70] W. Satuła, D. Dean, J. Gary, S. Mizutori, and W. Nazarewicz, Phys. Lett. B 407, 103 (1997).

[71] M. Bender, R. Bernard, G. Bertsch, S. Chiba, J. Dobaczewski, N. Dubray, S. A. Giuliani, K. Hagino, D. Lacroix, Z. Li, P. Magierski, J. Maruhn, W. Nazarewicz, J. Pei, S. Péru, N. Pillet, J. Randrup, D. Regnier, P.-G. Reinhard, L. M. Robledo, W. Ryssens, J. Sadhukhan, G. Scamps, N. Schunck, C. Simenel, J. Skalski, I. Stetcu, P. Stevenson, S. Umar, M. Verriere, D. Vretenar, M. Warda, and S. Åberg, J. Phys. G: Nucl. Part. 47, 113002 (2020). 